\title{
Potential role for PADI-mediated histone citrullination in preimplantation development
}

\author{
Rui Kan ${ }^{1}$, Mei Jin ${ }^{1}$, Venkataraman Subramanian², Corey P Causey ${ }^{3}$, Paul R Thompson ${ }^{2}$ and Scott A Coonrod ${ }^{*}$
}

\begin{abstract}
Background: The peptidylarginine deiminases (PADIs) convert positively charged arginine residues to neutrally charged citrulline on protein substrates in a process that is known as citrullination or deimination. Previous reports have documented roles for histone citrullination in chromatin remodeling and gene regulation in several tissue types, however, a potential role for histone citrullination in chromatin-based activities during early embryogenesis has not been investigated.

Results: In the present study, we tested by laser scanning confocal indirect immunofluorescence microscopy whether specific arginine residues on the histone $\mathrm{H} 3$ and $\mathrm{H} 4 \mathrm{~N}$-terminal tails $(\mathrm{H} 4 \mathrm{R} 33, \mathrm{H} 3 \mathrm{R} 2+8+17$, and $\mathrm{H} 3 \mathrm{R} 26)$ were citrullinated in mouse oocytes and preimplantation embryos. Results showed that all of the tested residues were deiminated with each site showing a unique localization pattern during early development. Given these findings, we next tested whether inhibition of PADI activity using the PADI-specific inhibitor, Cl-amidine, may affect embryonic development. We found that treatment of pronuclear stage zygotes with $\mathrm{Cl}$-amidine reduces both histone $\mathrm{H} 3$ and $\mathrm{H} 4$ tail citrullination and also potently blocks early cleavage divisions in vitro. Additionally, we found that the $\mathrm{Cl}$-amidine treatment reduces acetylation at histone $\mathrm{H} 3 \mathrm{~K} 9, \mathrm{H} 3 \mathrm{~K} 18$, and $\mathrm{H} 4 \mathrm{~K} 5$ while having no apparent effect on the repressive histone H3K9 dimethylation modification. Lastly, we found that treatment of zygotes with trichostatin A (TSA) to induce hyperacetylation also resulted in an increase in histone citrullination at $\mathrm{H} 3 \mathrm{R} 2+8+17$.

Conclusions: Given the observed effects of $\mathrm{Cl}$-amidine on embryonic development and the well documented correlation between histone acetylation and transcriptional activation, our findings suggest that histone citrullination may play an important role in facilitating gene expression in early embryos by creating a chromatin environment that is permissive for histone acetylation.
\end{abstract}

Keywords: Peptidylarginine deiminase, Citrullination, Histone modification, Preimplantation development, Cl-amidine

\section{Background}

The fundamental repeating unit of chromatin is the nucleosome that contains two superhelical turns of DNA wrapped around an octamer of two copies each of the core histones $\mathrm{H} 2 \mathrm{~A}, \mathrm{H} 2 \mathrm{~B}, \mathrm{H} 3$ and $\mathrm{H} 4$ [1,2]. Resolution of the nucleosome structure revealed that the $\mathrm{N}$-terminal histone tails protrude from the nucleosomal core in an unstructured manner [3] and contain an ever-growing number of posttranslational modifications such as acetylation, methylation, phosphorylation, and more recently, citrullination $[4,5]$. Importantly, these modifications, or "marks", play critical roles in many cellular functions, including DNA replication, condensation, and repair, as well as gene regulation [6]. Of

\footnotetext{
* Correspondence: sac269@cornell.edu

'Baker Institute for Animal Health, Cornell University, Ithaca, NY 14850, USA Full list of author information is available at the end of the article
}

these modifications, histone acetylation is perhaps most strongly associated with gene regulation. Increasing levels of histone acetylation are correlated with a transcriptionally permissive state whereas deacetylated histone are closely associated with transcriptional repression [7]. Histone acetylation is also implicated in the activation of embryonic gene expression in preimplantation embryos [8]. For example, previous reports investigating late two-cell embryos have found that inducing histone hyperacetylation with HDAC inhibitors stimulates global transcription [9] and depletion of HDAC1 by RNAi results in elevated levels of specific gene targets [8]. Previous studies in somatic cells have demonstrated that specific histone modifications can directly affect the levels of other marks and this interplay leads to a complex mechanism of gene regulation, frequently referred to as the "histone code" [10,11]. While

\section{Biomed Central}


fewer of these types of studies have been carried out in early embryos, several reports have found that cross-talk exist between histone acetylation and histone methylation in normal and cloned embryos [12].

PADI enzymes are increasingly being associated with the regulation of chromatin structure and gene activity via histone citrullination. For example, we have found that PADI4-mediated citrullination of histone $\mathrm{H} 4$ arginine 3 at the TFF1promoter in MCF7 cells appears to regulate the expression of this canonical estrogen receptor target [13]. Others have shown that PADI4-mediated histone citrullination plays a role in regulating other target genes such as TRP53 and OKL38 [14,15]. In addition to PADI4, we recently found that PADI2 localizes to the nucleus of mammary epithelial cells and appears to target histone $\mathrm{H} 3$ for citrullination [16], thus suggesting that multiple PADIs regulate chromatin-based activities.

We have previously documented that oocyte -andembryo abundant PADI6 is required for female fertility, with PADI6-null embryos arresting at the two-cell stage of development [17]. Given the growing body of literature linking PADI enzymes to histone citrullination and the abundance of PADI6 in oocytes and early embryos, we first tested whether histones were citrullinated in oocytes and preimplantation embryos. Next, we treated embryos with the PADI-specific inhibitor, $\mathrm{Cl}$-amidine, to confirm that the observed citrulline marks were generated by PADI activity and also to test whether inhibition of PADI activity may affect preimplantation development in vitro. Lastly, to gain insight into potential mechanisms by which histone citrullination may regulate gene activity, we tested whether inhibition of PADI activity in early embryos affects histone acetylation and whether induction of histone hypoacetylation affected levels of histone citrullination. Findings from this study are discussed below.

\section{Results and discussion}

Citrullination of histone $\mathrm{H} 3$ and $\mathrm{H} 4$ tails in oocytes and in preimplantation embryos appears to be robust and dynamic

In the present study, the status of histone $\mathrm{H} 3$ and $\mathrm{H} 4$ citrullination during early development was investigated by staining fully grown mouse germinal vesicle (GV) stage oocytes and preimplantation embryos with three site-specific citrullinated histone antibodies: anti-histone H4 citrulline 3 (H4Cit3), anti-histone H3 citrulline 2, 8, and $17(\mathrm{H} 3 \mathrm{Cit} 2+8+17)$ and anti-histone $\mathrm{H} 3$ citrulline 26 (H3Cit26) $[13,16,18]$. Results from these confocal indirect immunofluorescence studies showed that H4Cit3 staining was observed in the nucleus and cytoplasm of oocytes and early embryos at interphase. Interestingly, the strongest staining for this citrulline modification appears to occur on mitotic metaphase chromatin, suggesting that $\mathrm{H} 4 \mathrm{Cit} 3$ may play a role in chromatin condensation or decondensation at metaphase (Figure 1A). Staining with the anti-H3Cit $2+8+17$ antibody found that this modification also stained nuclei during interphase (Figure 1B). However, at metaphase, this antibody appeared to primarily stain the spindle apparatus (Figure 1B; arrows in the panel of anaphase II) and a structure at opposed regions of two cell blastomeres that appears to be the microtubule-containing mid-body $[19,20]$ (Figure 1B; arrowheads in panels of 2cell). Interestingly, in somatic cells, an anti-phospho-H3 serine 10 antibody detected histones with this modification at the anaphase spindle apparatus and at the midbody during cytokinesis [21]. These investigators speculated that this modification may mark histones for removal from the nucleosome or possibly that this modified histone may actually play a direct role in cytokinesis by functioning at the mid-body [22]. It is currently not known whether citrullinated histones in the cytoplasm of oocytes and early embryos might have a similar function. Alternatively, however, it is also possible that the observed spindle and mid-body staining with the $\mathrm{H} 3 \mathrm{Cit} 2+8+17$ antibody is non-specific in nature.

The staining pattern observed with the anti-H3Cit26 was perhaps the most interesting of all. Results found that both oocytes and early embryos showed a strong punctate cytoplasmic signal that appeared to coalesce into larger aggregates as the embryos developed (Figure 1C) and these foci appeared by light microscopy to be lipid droplets. To test this hypothesis we stained mutant MATER GV oocytes (which have elevated levels of lipid droplets [18]) with Nile Red or with the H3Cit26 antibody. Results show (Additional file 1) that the H3Cit26-containing cytoplasmic foci clearly appear to be lipid droplets. Interestingly, a recent study found that lipid droplets in Drosophila embryos are maternallyderived and that these structures contain $\sim 50 \%$ of all embryonic histones. This finding suggests that the lipid droplets function to sequester maternal histones in the early embryo until they are needed for chromatin-based activities [19]. An intriguing possibility is that the H3Cit26 modification marks histones for lipid droplet storage and/or possibly shuttling histones between lipid droplets and the nucleus. H3Cit26 staining within the nucleus was also interesting; whereas little to no signal was seen in GV stage oocytes, strong staining was observed on the outer margins of both male and female pronuclei and around the nucleoli of two-cell embryos (Figure 1C; arrows in panels of 2-PN and 2-cell). By the four-cell stage of development no nuclear staining was observed. Given that embryonic genome activation is known to initiate at the late pronuclear/early two-cell stage, this observation raises the possibility that this particular citrulline modification may play a role in 


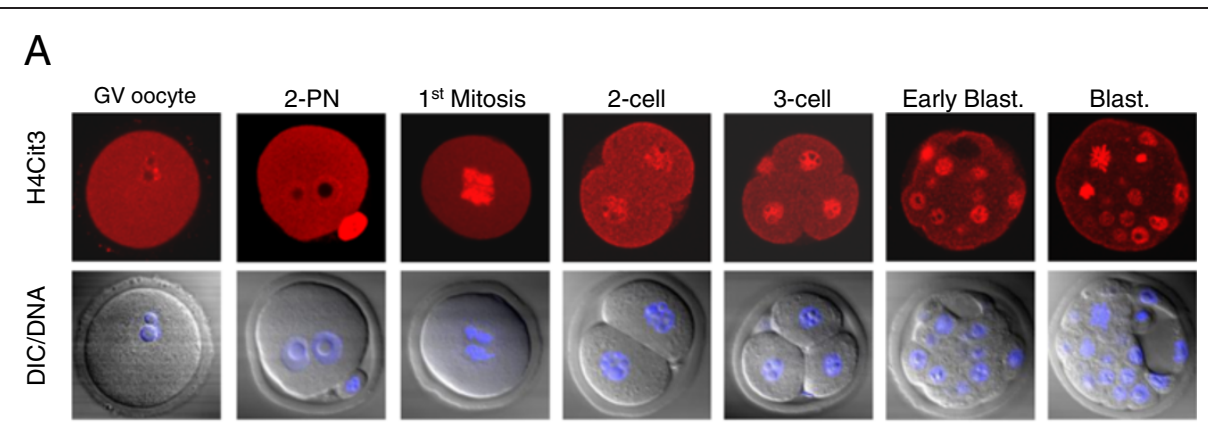

B
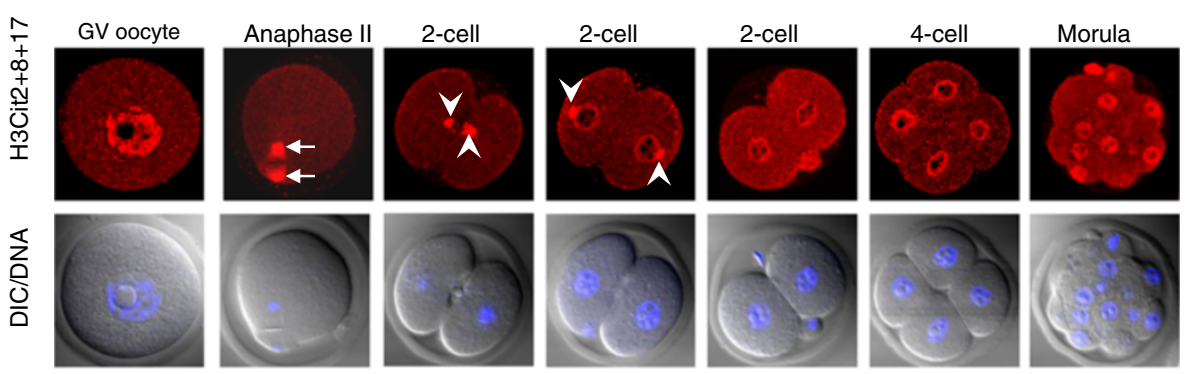

C
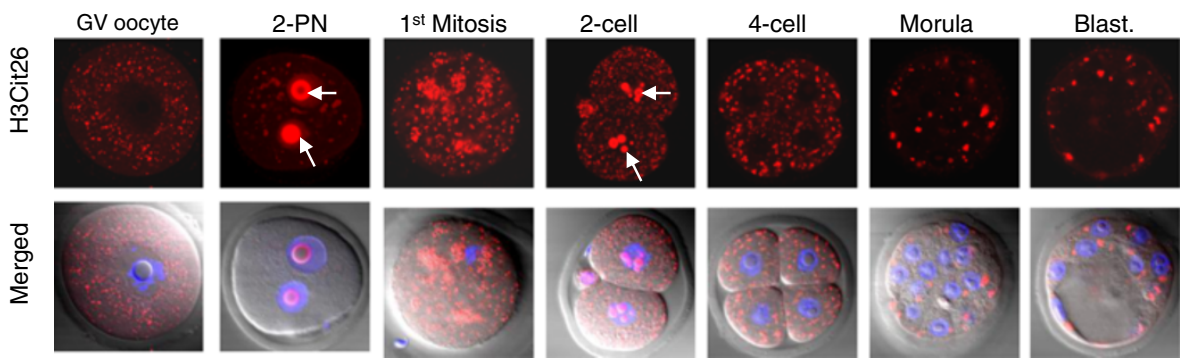

Figure 1 Temporal and spatial distribution of histone $\mathrm{H} 3$ and $\mathrm{H} 4$ citrullination in oocytes and preimplantation embryos. A. Oocytes and embryos were isolated from CD1 female mice, fixed, permeabilized, and immunolabeled with antibodies against H4Cit3 (red). B. Same as (A) except anti-H3Cit2 $+8+17$ antibodies (red) were used. Spindle apparatus and microtubule based mid-bodies are indicated by arrows and arrowheads, respectively. C. Same as (A) except anti-H3Cit26 antibodies (red) were used. Nucleoli are denoted by arrows. All samples were counterstained with 4',6-diamidino-2-phyenylindole (DAPI) to visualize DNA (Blue). Cells were imaged by laser scanning confocal microscopy. DIC, differential interference contrast. PN, pronuclear. Blast., blastocyst.

activation of the embryonic genome. Taken together, our data raise the possibility that the different histone modification sites may play different roles in preimplantation development.

Given that each of these anti-citrullinated histone antibodies showed both cytoplasmic and nuclear localization patterns, we next confirmed the specificity of our antibodies by testing whether pre-absorption of the antibodies with their cognate peptide affected indirect immunofluorescence signal intensity levels (Additional file 2). Results showed that peptide preabsorption suppressed the fluorescence intensity for each of the three antibodies (Additional files $2 \mathrm{~A}, 2 \mathrm{~B}$, and $2 \mathrm{C}$, respectively). These results suggest that the localization patterns observed for the H4Cit3, $\mathrm{H} 3 \mathrm{Cit} 2+8+17$, and H3Cit26 are specific.

\section{$\mathrm{Cl}$-amidine blocks mouse embryonic development} beyond the two to four cell stage in vitro

The above observations suggested that PADI-mediated histone citrullination may play an important, previously unknown, role in early development. Given that PADI6 is essential for early cleavage divisions, we next tested whether levels of these modifications were reduced in PADI6-null mouse oocytes/early embryos. We found that loss of PADI6 did not appear to affect histone citrullination levels (see Additional file 3A, 3B, and 3C). Given PADI4's previously documented roles in histone citrullination and gene regulation $[13,14]$, we then tested citrullinated histone levels in PADI4-null oocytes. Again, we did not observe any appreciable loss in levels of citrullinated histone in this mutant line (see Additional 
file $4 \mathrm{~A}$ and $4 \mathrm{~B})$. Together, these observations suggest, neither PADI4 nor PADI6 catalyze these specific citrulline modifications on histones in oocytes or early embryos.

Given these observations, and the lack of mutant PADI1, PADI2, and PADI3 mouse lines, we next decided to test the effects of a newly developed PADI inhibitor, $\mathrm{Cl}$-amidine, on histone citrullination and on early embryonic development. Cl-amidine has been shown to irreversibly block the activity of all PADI enzymes in vitro and has also been shown in cell culture and mouse-based assays to functionally inhibit PADI activity in vivo [20-22]. We first tested whether Clamidine suppressed citrulline levels on histones in early embryos using the H4Cit3, H3Cit2 + 8+17, and H3Cit26 antibodies. PN zygotes were cultured in KSOM media supplemented without or with $250 \mu \mathrm{M}$ of $\mathrm{Cl}$-amidine. Embryos at the 4-cell stage from $\mathrm{KSOM}$ and $\mathrm{Cl}$-amidine groups were fixed after being cultured for $\sim 42$ hours and $\sim 68$ hours, respectively, to ensure developmental arrest at cleavage stage. Next, the embryos were stained with the anti-citrullinated histone antibodies and then evaluated by laser scanning confocal microscopy. Results showed that staining levels for the $\mathrm{H} 4 \mathrm{Cit} 3$ and $\mathrm{H} 3 \mathrm{Cit} 2+8+17$ antibodies was reduced, compared with the KSOM control group (Figure 2A and $2 \mathrm{~B}$, arrows in top panels). Interestingly, however, Clamidine treatment did not appear to affect levels of the H3Cit26 modification, suggesting that histone citrullination at this site may have occurred in oocytes prior to drug treatment (Figure 2C). Actin levels and localization did not appear to be affected by $\mathrm{Cl}$-amidine. These results support the hypothesis that the histone citrullination in embryos is catalyzed by PADI activity.

We next investigated the effects of $\mathrm{Cl}$-amidine on embryonic development in vitro. As a control for these experiments, we also tested the effect of $\mathrm{H}$-amidine on development. This analog displays very weak PADI inhibitory activity [31] with, for example, the IC50 values of $\mathrm{Cl}$ amidine and $\mathrm{H}$-amidine for PAD4 inhibition in vitro being $5.9 \mu \mathrm{M}$ and $>1000 \mu \mathrm{M}$, respectively [31]. The structures of these two compounds are shown in Figure 2D and 2E. PN stage zygotes were cultured for $\sim 68$ hours in KSOM media supplemented with $250 \mu \mathrm{M}$ of either $\mathrm{Cl}$-amidine or $\mathrm{H}$ -

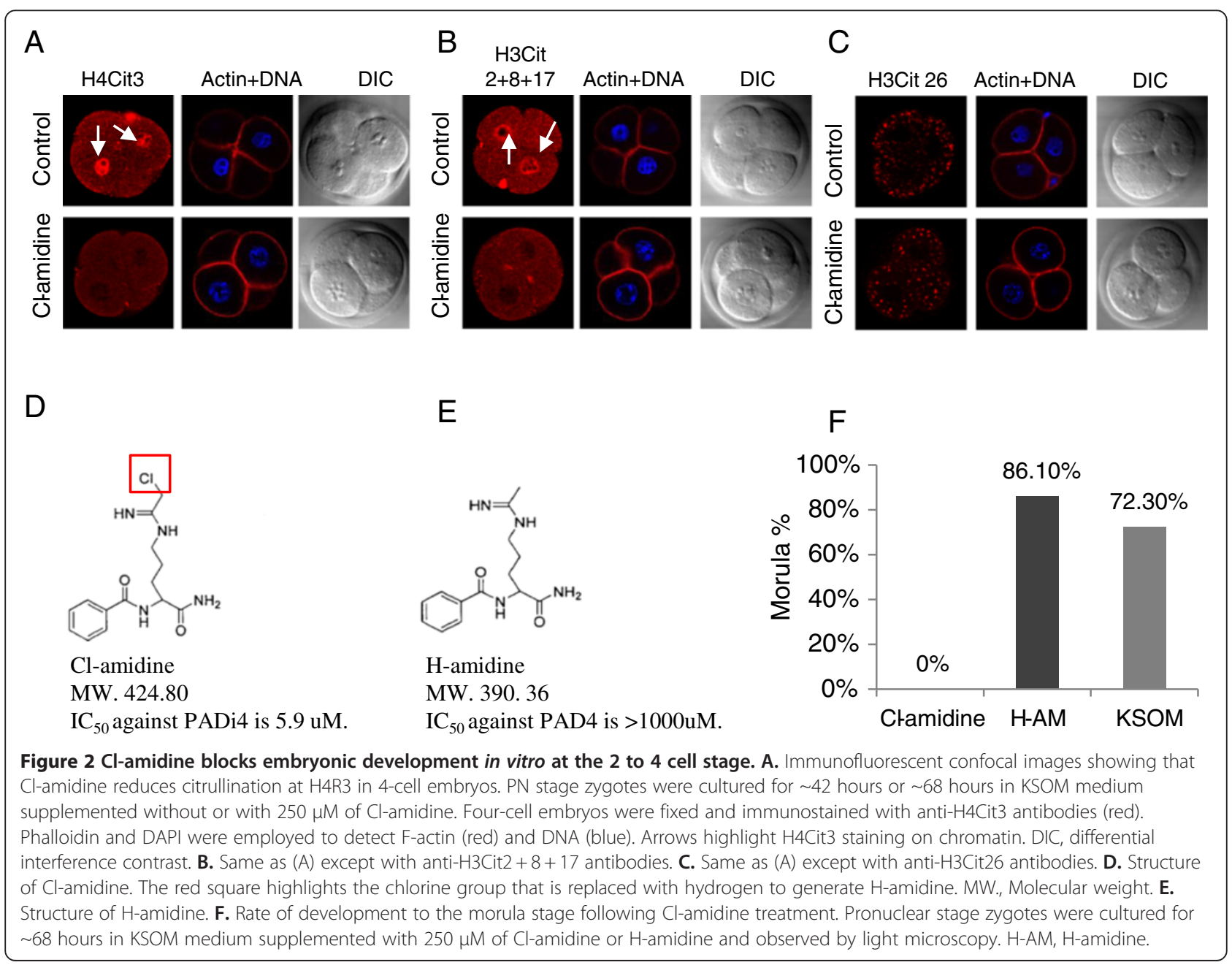


amidine. The number and developmental stage of embryos was then evaluated using light microscopy. Results showed that embryos arrested either at the 2-4 cell stage $(83 \%$, $\mathrm{n}=100)$ or at the 1 -cell stage $(17 \%, \mathrm{n}=100)$ in the $\mathrm{Cl}$ amidine group, while $86.1 \%$ of embryos $(\mathrm{n}=94)$ in the $\mathrm{H}$ amidine group and $72.3 \%$ of embryos $(\mathrm{n}=36)$ in $\mathrm{KSOM}$ medium alone developed to the morula stage (Figure 2F and Table 1). We note here that (1) the concentration of $\mathrm{Cl}$-amidine used in our study is within the range of that used to functionally block PADI activity in somatic cells $[14,23]$ and that (2) lower concentrations of $\mathrm{Cl}$-amidine did not affect embryonic development (Additional file 5A and $5 \mathrm{~B})$. Our finding that $\mathrm{Cl}$-amidine suppressed histone citrullination in cleavage-stage embryos suggested that the observed effects of $\mathrm{Cl}$-amidine on development were due to specific inhibition of PADI activity. However, it is also possible that the inhibitor blocked development because of non-specific toxic side-effects. To address this possibility, we first examined embryo viability following $\mathrm{Cl}$-amidine and $\mathrm{H}$-amidine treatment using the vital dye propidium iodide (PI). Results showed that nuclei from both $\mathrm{Cl}-$ amidine and $\mathrm{H}$-amidine treated $(250 \mu \mathrm{M})$ embryos were not stained with PI $(20 \mu \mathrm{g} / \mathrm{ml})$ while nuclei from embryos that were treated with $\mathrm{Cl}$-amidine and extracted with $0.1 \%$ Triton were strongly stained with PI (Additional file 6A). These results indicate that the plasma membrane of $\mathrm{Cl}-$ amidine and $\mathrm{H}$-amidine treated embryos appeared functional. To further confirm embryo viability, we next evaluated the mitochondrial membrane potential of $\mathrm{Cl}$-amidine and $\mathrm{H}$-Amidine treated embryos using the JC-1 fluorescent dye, which accumulates in functional mitochondria as redstaining aggregates (Additional file 6B). Results showed that the mitochondrial membrane potential appeared to be similar between the $\mathrm{Cl}$-amidine and $\mathrm{H}$-amidine treatment groups, suggesting the $\mathrm{Cl}$-amidine does not affect mitochondrial health. Together, these findings suggest that PADI activity is required for progression of embryonic development beyond the two to four cell stage.

Treatment of embryos with C-amidine suppresses histone $\mathrm{H} 3$ and $\mathrm{H} 4$ acetylation while having no apparent effect on the repressive H3K9 Di-methyl modification

As noted, histone acetylation is well correlated with activation of gene expression in somatic cells and is also believed to play an important role in modulating gene

Table 1 The effect of $\mathrm{Cl}$-amidine on early embryonic development

\begin{tabular}{llccc}
\hline Group & No. & \multicolumn{3}{c}{ Stage of embryos (\%) } \\
\cline { 3 - 5 } & & 1 Cell & 2-4 Cell & Morula \\
\hline Cl-amidine & 100 & $17(17)$ & $83(83)$ & $0(0)$ \\
H-amidine & 36 & $5(13.9)$ & $0(0)$ & $31(86.1)$ \\
KSOM & 94 & $22(23.4)$ & $4(4.3)$ & $68(72.3)$ \\
\hline
\end{tabular}

expression in preimplantation embryos [7-9]. In order to begin testing whether histone citrullination may play a role regulating gene activity in early embryos, we tested whether suppression of histone citrullination with $\mathrm{Cl}$ amidine affected levels of histone acetylation on $\mathrm{H} 3$ and $\mathrm{H} 4$ tails. Results showed that $\mathrm{Cl}$-amidine treatment significantly reduced levels of histone $\mathrm{H} 4$ acetylation (Figure 3A and 3E). The fluorescent intensities for hyper acetylated H4 (hyper acH4) and H4K5 Acetyl (H4acK5) in $\mathrm{Cl}$-amidine, TSA, and KSOM groups were presented in Figure 3B and 3F. Additionally, $\mathrm{Cl}$-amidine also dramatically reduced the level of acetylation on the $\mathrm{H} 3$ tail, namely H3K9 Acetyl (H3acK9) and H3K18 Acetyl (H3acK18) (Figure 3A and 3C). The fluorescent intensities for these modifications were showed in Figure 3B and 3D. To gain further insight into the mechanisms by which histone citrullination may regulate gene activity, we also tested whether $\mathrm{Cl}$-amidine affected levels of dimethylated histone $\mathrm{H} 3 \mathrm{~K} 9$, a modification closely associated with transcriptional repression [24]. We found that levels of this modification were not significantly affected by $\mathrm{Cl}$-amidine treatment (Figure $3 \mathrm{C}$ and $3 \mathrm{D}$ ). To validate the specificity of the acetylated histone antibodies, the embryos were also treated with the HDAC inhibitor Trichostatin A (TSA), and as expected, staining for acetylated histones (hyper acH4, H4acK5, H3acK9, and H3ack18) was elevated following TSA treatment (Figure 3). Taken together, these results suggest that inhibition of PADI-mediated histone citrullination suppresses histone acetylation.

\section{Histone hyperacetylation promotes histone citrullination in early embryos}

To further explore the potential interplay between histone citrullination and acetylation, the colocalization of citrullination at $\mathrm{H} 3 \mathrm{R} 2+8+17$ and acetylation at $\mathrm{H} 3 \mathrm{~K} 9$ was tested in 2-cell embryos. Results revealed that these two histone modifications localize, in part, to different regions of the nucleus, and they do appear to colocalize at specific foci in 2-cell embryos (Figure 4A, arrows highlight overlapping regions which are seen as yellow in the overlay). To further test whether there was a potential crosstalk between histone citrullination and acetylation, levels of H3R $2+8+17$ citrullination were examined in embryos treated with either TSA or $\mathrm{Cl}$-amidine. To perform this experiment, $\mathrm{PN}$ zygotes were recovered from B6D2F1/J females and cultured in KSOM medium supplemented with either $100 \mathrm{nM}$ of TSA or $250 \mu \mathrm{M}$ of Cl-amidine, respectively, for $\sim 68$ hours. Results showed that, as expected, levels of H3Cit2 + $18+17$ were significantly reduced following $\mathrm{Cl}$-amidine treatment (Figure 4B-C). Interestingly, however, we found that induction of histone hyperactylation via TSA treatment also resulted in significantly increased levels of citrullination at $\mathrm{H} 3 \mathrm{R} 2+8+17$ (Figure 4B-C). This observation further 


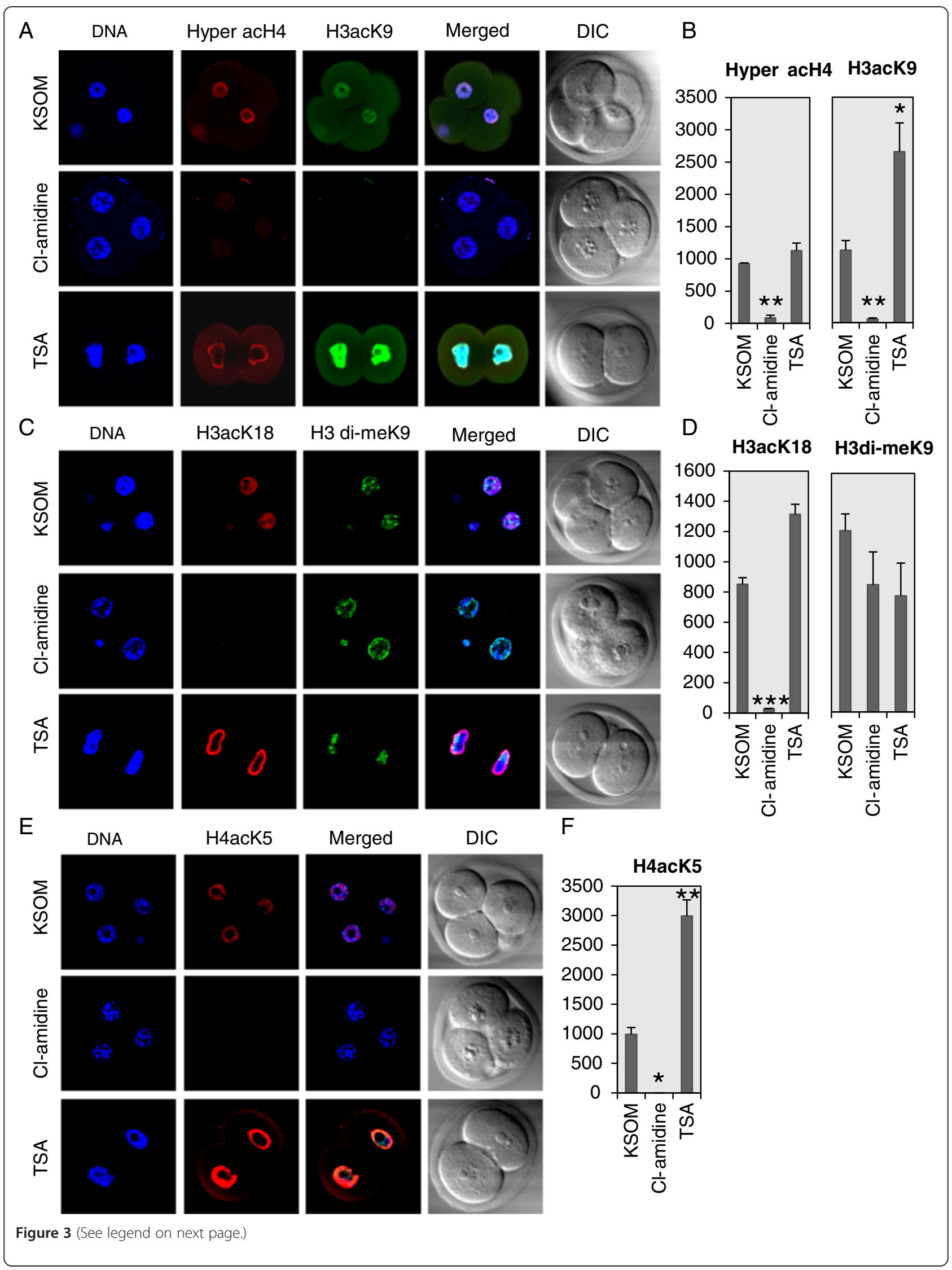


(See figure on previous page.)

Figure 3 Effect of $\mathrm{Cl}$-amidine and TSA treatment on histone $\mathrm{H} 3$ and $\mathrm{H} 4$ acetylation and methylation. A. PN zygotes were cultured for $\sim 68$ hours in KSOM medium supplemented with either $250 \mu \mathrm{M}$ of $\mathrm{Cl}$-amidine or $100 \mathrm{nM}$ of TSA. Two or four-cell embryos were fixed and immunostained with antibodies to hyperacetylated H4 (red) and acetyl H3K9 (green). DAPI was utilized to detect DNA (blue). DIC, differential interference contrast. B. Histograms documenting the fluorescent intensity of the hyperacetylated $\mathrm{H} 4$ and acetyl H3K9 signals shown in A. C. Same as (A) except antibodies against acetyl H3K18 (red) and di-methyl H3K9 (green) were used. D. Histograms documenting the fluorescent intensity shown in B experiments. E. Same as (A) except antibodies against acetyl H4K5 (red) were used. F. Histogram documenting the fluorescent intensity in experiment shown in E. Data are presented as mean + SEM. ${ }^{*} P<0.05,{ }^{* *} P<0.005,{ }^{* *} P<0.001$ (two-tailed paired Student's t-test).

suggests that there is a reinforcing relationship between acetylation and citrullination and further highlights the potential interplay between these two modifications on chromatin in preimplantation mammalian embryos.

\section{Conclusions}

This report is the first to document the presence of citrullinated histones in mammalian oocytes and preimplantation embryos. The use of three site-specific citrullinated histone antibodies found that histone citrullination is likely playing several unique, yet to be defined roles on chromatin templated events. We found that the PADI inhibitor, $\mathrm{Cl}$-amidine, potently blocks embryonic development beyond the 4-cell stage, thus further highlighting the important role of PADIs in early development. This observation also raises the possibility that PADI inhibitors could potentially be utilized as novel contraceptives. Our study also showed that $\mathrm{Cl}$-amidine specifically suppressed histone acetylation on the $\mathrm{H} 3$ and $\mathrm{H} 4$ tails while not affecting levels of the transcriptionally repressive histone H3K9 dimethyl modification. Further, we found that induction of histone hyperacetylation leads to enhanced histone citrullination. Mechanistically, as with numerous other histone modifications [10,11], these observations raise the distinct possibility that

A

DNA

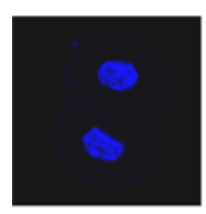

B
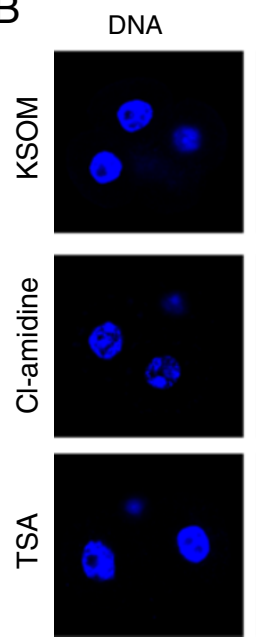

H3Cit $2+8+17$

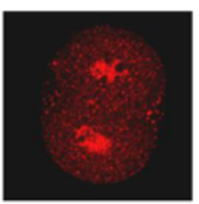

H3Cit $2+8+17$
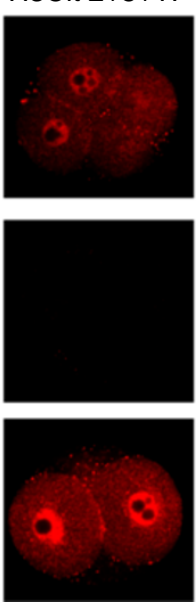

H3acK9

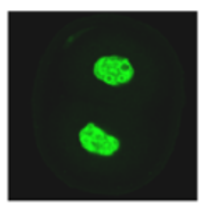

Merged
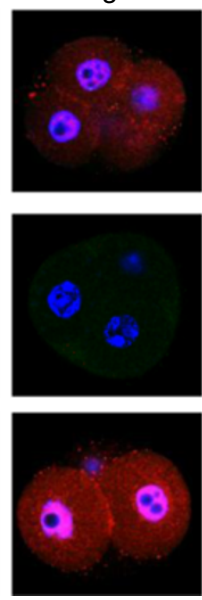

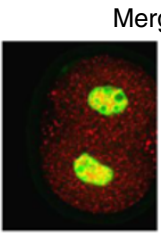

Merge
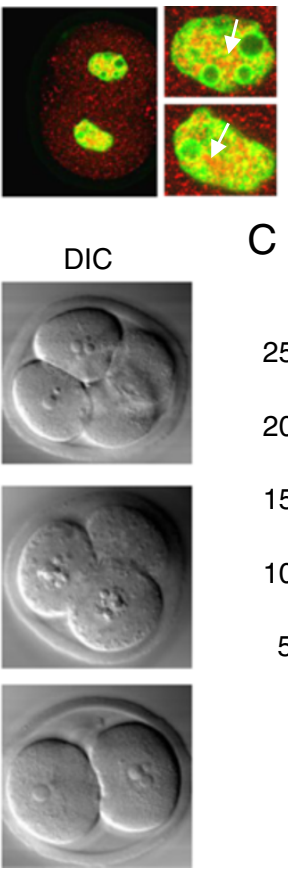

C

DIC
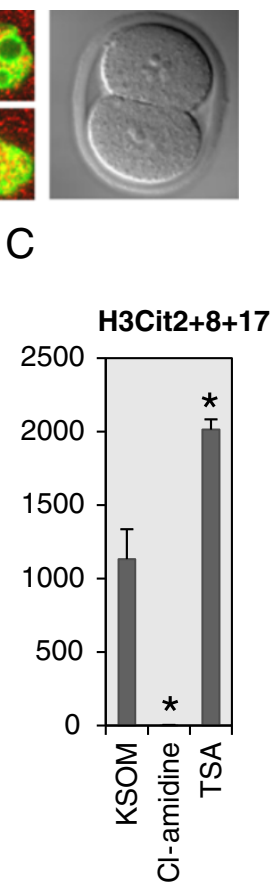

Figure 4 Potential crosstalk between histone citrullination and acetylation in early embryos. A. Colocalization of citrullinated H3R2 + 8 17 and acetylated H3K9. Two-cell embryos were immuno-stained with antibodies to H3Cit2 + 8+ 17 (red) and H3acK9 (green). The colocalization is highlighted in yellow in the merged panel. B. PN stage zygotes were co-cultured for $\sim 68$ hours in $\mathrm{KSOM}$ medium with $250 \mu \mathrm{M}$ of $\mathrm{Cl}$-amidine or $100 \mathrm{nM}$ of TSA. Two or four-cell embryos were fixed and immuno-stained with antibodies to H3Cit $2+8+17$ (red). DAPI was utilized to detect DNA (blue). DIC, differential interference contrast. Data are presented as mean + SEM. ${ }^{*} P<0.05$, (two-tailed paired Student's $t$ test). 
the citrulline modification on histones may function as a "platform" for binding by histone acetyltransferases (HATs), thus facilitating transcriptional activation by enhancing levels of histone acetylation. More detailed studies are now required to test this hypothesis. We predict that outcomes from the current study will likely lead to new and important insight into epigenetic regulation of the oocyte to embryo transition.

\section{Methods}

\section{Animals}

The generation of mouse mutants and genotyping strategies for the Padi6 and Mater null strain has been described previously $[17,25]$. To generate Padi4-null mice, the entire genomic sequence of Padi4 was replaced in frame with the coding sequence of LacZ and a Lox-flanked neomycin gene driven by PGK-EM7 promoter. B6D2F1/J and CD-1 mice were purchased from the Jackson Laboratory and Charles River Laboratories, respectively. All mice were housed in the Cornell University Animal Facility (Ithaca, NY) and procedures using these mice were reviewed and approved by the Cornell University Institutional Animal Care and Use Committee. Studies were performed in accordance with the Guiding Principles for the Care and Use of Laboratory Animals.

\section{Oocyte and embryo collection}

All experiments were performed using B6D2F1/J and CD-1 female mice (age 4-8 weeks) primed with gonadotrophins (Sigma) to obtain fully-grown GV oocytes, ovulated oocytes, and embryos (following mating with CD1 males). All oocytes and embryos were collected in M2 medium (Sigma) unless otherwise stated. Culture medium was supplemented with $25 \mathrm{mM}$ of milrinone (Sigma) to inhibit GVBD. Embryos at different developmental stages were collected and processed at different times.

\section{Immunofluorescence and laser scanning confocal microscopy}

Indirect immunofluorescence labeling confocal microscopy was undertaken as described previously [26]. Rabbitanti-H4Cit3 (1:50, Abcam), rabbit-anti-H3Cit2 + 8+ 17 (1:50, Abcam), rabbit-anti-H3Cit26 (1:50, Abcam), rabbitanti-hyper acetyl H4 (1:50, Millipore), mouse-anti-acetyl H3K9 (1:20, Abcam), rabbit-anti-acetyl H4K5 (1:50, Abcam), rabbit-anti-acetyl H3K18 (1:50, Abcam), and mouse-anti-dimethyl H3K9 (1:20, Abcam) antibodies were used for this study. Images were obtained on LSM 510 laser scanning confocal microscopy (Carl Zeiss, Germany) equipped with Zen 2007 software for image processing. In the multicolor labeling experiments, the confocal configuration was set up to avoid the bleed-through of fluorescence dyes. To test for bleed-through, oocytes were stained with each dye separately and images were taken with multiple channels. Each signal was found to be well resolved from other signals. Images for each developmental series were collected under similar conditions using the following settings: $\mathrm{ex}=555 \mathrm{~nm}$, em $=565 \mathrm{~nm}$, laser power $12 \%$, frame size $1024 \times 1024$, scanning speed 7 , averaging number 4, detector gain around 700, digital offset around -15, and Zen 2009 software. To directly compare changes in signal intensity for embryos from different treatment groups, confocal images were acquired under an identical condition for those samples.

\section{Embryo culture and drug treatment}

The KSOM medium for embryo culture has been described elsewhere [27].B6D2F1/J females were primed with 10 IU PMSG (Sigma) followed by 10 IU hCG (Sigma) injection, then housed with CD1 males. PN zygotes were collected in M2 medium at $\sim 26$ hours post hCG and cultured in KSOM for $\sim 42$ hours or 68 hours supplemented with $250 \mu \mathrm{M}$ of Cl-amidine, $250 \mu \mathrm{M}$ of $\mathrm{H}$-amidine, or with $100 \mathrm{nM}$ of TSA (Sigma). Embryos cultured at $37{ }^{\circ} \mathrm{C}$ in an atmosphere of $5 \% \mathrm{CO}_{2}, 5 \% \mathrm{O}_{2}$ and $90 \% \mathrm{~N}_{2}$ were fixed and immunostained with antibodies at different time points for analyses. Digital images were recorded on the confocal microscopy.

\section{Citrulline antibody absorption assay by antigen peptide}

All antibodies and antigen peptides were purchased from Abcam with the exception of the H4Cit3 peptide which was a kind gift from David Allis at Rockefeller University. The ratios of antibody and peptide for $\mathrm{H} 4 \mathrm{Cit} 3$, H3Cit $2+8+$ 17 , and $\mathrm{H} 3 \mathrm{Cit} 26$ were $1 \mathrm{~mol}: 20$ mols, $1 \mu \mathrm{l}: 6 \mu \mathrm{ls}$, and 1mol:40mols, respectively. The antibody and peptide were added to the antibody dilution buffer (1\% normal goat serum, $0.5 \% \mathrm{BSA}$ in $\mathrm{PBS}$ ) and incubated on a rotator at room temperature for 2 hours for the H4Cit3 and $\mathrm{H} 3 \mathrm{Cit} 2+$ $8+17$ mixtures or for 1 hour $\left(4^{\circ} \mathrm{C}\right)$ for the $\mathrm{H} 3 \mathrm{Cit} 26$ mixture.

Deionized water replaced the histone modification peptides as a control. GV-oocytes or 2-cell embryos were collected from CD1 females at $\sim 46$ hours post PMSG and $\sim 46$ hours post hCG and processed for immunofluorescence and laser scanning confocal microscopy as described above.

\section{Nile red staining of mouse oocytes}

Nile red powder (Sigma) was dissolved in DMSO to give a stock solution of $1 \mathrm{mg} / \mathrm{ml}$ and stored at $-20^{\circ} \mathrm{C}$. GV-oocytes were collected in M2 media supplemented with IBMX from Mater mutant females. After three quick washes in PBS/PVA, oocytes were transferred into $4 \%$ paraformaldehyde/PBS and incubated for $30 \mathrm{~min}$ at room temperature. Oocytes were briefly washed three times in PBS/PVA again and transferred into nile red working solution $(1 \mu \mathrm{g} / \mathrm{ml})$ for 
30 min following the fixation. After the nile red staining, oocytes were washed three times and carefully added to the drop of Slowfade Gold antifade reagent (Molecular probe) on slides, and then a cover slide was placed on top of the drop. Nile red fluorescence was captured by Laser Scanning Confocal microscopy (Carl Zeiss).

\section{Analysis of embryo viability}

Pronuclear stage zygotes were retrieved from B6D2F1/J females at $\sim 26$ hours post hCG and cultured for $\sim 68$ hours in KSOM medium supplemented with $250 \mu \mathrm{M}$ of $\mathrm{Cl}$ amidine or H-AM. Embryos from these two groups were then incubated with $20 \mu \mathrm{g} / \mathrm{ml}$ of PI (Sigma) in KSOM for 5 min at $37{ }^{\circ} \mathrm{C}$ in an atmosphere of $5 \% \mathrm{CO}_{2}$, washed 3 times, and images were recorded using Zeiss epifluorescence microscopy (Carl Zeiss, Germany). A sub set of $\mathrm{Cl}$-amidine treated embryos were permeablized with $0.1 \%$ Triton for 20 min prior to PI staining to serve as positive control.

JC-1 staining of cultured embryos was performed according to the Invitrogen protocol. Briefly, embryos were stained with $10 \mu \mathrm{g} / \mathrm{ml}$ of $\mathrm{JC}-1$ (Invitrogen) in $\mathrm{KSOM}$ for $10 \mathrm{~min}$ at $37^{\circ} \mathrm{C}$ in an atmosphere of $5 \% \mathrm{CO}_{2}$, washed 3 times with $\mathrm{KSOM}$, and images were captured by Zeiss epifluorescence microscopy using fluorescein isothiocyanate (FITC) and tetramethyl rhodamine isothiocyanate (TRITC) filters.

\section{Statistical analysis}

All samples used for quantification included at least four nuclei. Data were compiled and analyzed using Microsoft Excel 2007. Comparisons were made for the intensity of histone modifications in embryos cultured in KOSM media supplemented with or without $\mathrm{Cl}$-amidine using unpaired $t$-test. The threshold of statistical significance was set at $P<0.05$.

\section{Additional files}

Additional file 1: Localization of $\mathrm{H} 3 \mathrm{Cit} 26$ to lipid droplets in mutant Mater GV stage oocytes. Mutant Mater oocytes (which contain high levels of lipid droplets) were probed with antibodies to H3Cit 26 (A) and the oocytes were also stained with Nile Red (B). Confocal images were taken of the stained oocytes following counterstaining with DAPI to visualize DNA (blue). DIC, differential interference contrast.

Additional file 2: Peptide pre-absorption assay to test for antibody specificity. Confocal images were taken of CD1 GV-stage oocytes stained with H4Cit3 (A), H3Cit 2+8+17 (B) antibodies and 2-cell embryos

stained with $\mathrm{H} 3 \mathrm{Cit} 26$ (C) antibodies. In top panels, deionized water was used instead of the peptide solution as a control. In the bottom panels, anti-citrullinated histone antibodies were pre-absorbed with cognate peptides prior to staining oocytes and embryos. Oocytes/embryos were counterstained with DAPI to visualize DNA (blue). DIC, differential interference contrast.

Additional file 3: Comparison of citrullination levels at H3R2 + 8 + 17, H4R3, and H3R26 in PADI6 wild-type and null oocytes. Confocal images were taken of wild-type and PADI6-null GV stage oocytes that had been probed with antibodies to H3Cit 2+8+17 (A), H4Cit3 (B), and H3Cit26 (C). Oocytes were counterstained with
DAPI to visualize DNA (blue). DIC, differential interference contrast. Additional file 4: Comparison of citrullination levels at $\mathrm{H} 3 \mathrm{R} 2+8+17$ and H4R3 in PADI4 wild-type and null oocytes. Confocal images were taken of wild-type and PADI4 null GV stage oocytes that had been probed with antibodies to $\mathrm{H} 3 \mathrm{Cit} 2+8+17(\mathbf{A})$ and H4Cit3 (B). Oocytes were counterstained with DAPI to visualize DNA (blue). DIC, differential interference contrast.

Additional file 5: Titration of $\mathrm{Cl}$-amidine concentration for mouse embryo culture study. A. Pronuclear stage zygotes were cultured for $\sim 68$ hours in KSOM medium supplemented without or with 500 nM, 5 $\mu \mathrm{M}$, or $250 \mu \mathrm{M}$ of $\mathrm{Cl}$-amidine and images of the embryos were recorded by light microscopy. Images were taken in 40X magnification with DIC and the scale bar is $50 \mu \mathrm{m}$. B. Histogram showing the rate of embryonic development for the different treatment groups in $(A) .(n)=$ total number of embryos for each group. DIC, differential interference contrast.

Additional file 6: Assessment of embryo viability following $\mathrm{Cl}$ amidine treatment. Pronuclear stage zygotes were cultured for $\sim 68$ hours in $\mathrm{KSOM}$ medium supplemented with $250 \mu \mathrm{M}$ of $\mathrm{Cl}$-amidine or $\mathrm{H}$ amidine prior to the staining. A. Propidium iodide (PI) staining of cultured embryos. Embryos were stained with $20 \mu \mathrm{g} / \mathrm{ml}$ of PI in KSOM for $5 \mathrm{~min}$ and images were recorded by epifluorescence microscopy. Clamidine treated embryos were treated with $0.1 \%$ Triton for 20 min prior to PI staining and utilized as positive control to show the nuclear staining of nonviable cells. Propidium iodide, PI. B. JC-1 staining of embryos following treatment with $\mathrm{Cl}$-amidine or $\mathrm{H}$-amidine. Embryos were stained with $10 \mu \mathrm{g} / \mathrm{ml}$ of JC- 1 in $\mathrm{KSOM}$ for $10 \mathrm{~min}$ and fluorescence was captured by epifluorescence microscopy. DIC, differential interference contrast.

\section{Authors' contributions}

RK participated in the design of the study, performed experiments, and wrote the paper. MJ performed the mouse embryo culture experiment with Cl-amidine. SAC conceived, designed and coordinated the study, and also helped to write the paper. VS, CPC, and PRT contributed the PADI inhibitors and helped to coordinate the study. All authors read and approved the final manuscript.

\section{Acknowledgements}

This research was supported by NICHD grants RO1 38353 and RO3 522241 (S.A.C.) and by NIGMS grant GM079357 (P.R.T).

\section{Author details}

'Baker Institute for Animal Health, Cornell University, Ithaca, NY 14850, USA. 2Department of Chemistry, The Scripps Research Institute, 130 Scripps Way, Jupiter, FL 33458, USA. ${ }^{3}$ Department of Chemistry, University of North Florida, Jacksonville, FL 32224, USA.

Received: 6 December 2011 Accepted: 23 May 2012 Published: 19 June 2012

\section{References}

1. Wysocka J, Allis CD, Coonrod S: Histone arginine methylation and its dynamic regulation. Front Biosci 2006, 11:344-355.

2. Richmond TJ, Davey CA: The structure of DNA in the nucleosome core. Nature 2003, 423:145-150.

3. Luger K, Mader AW, Richmond RK, Sargent DF, Richmond TJ: Crystal structure of the nucleosome core particle at $2.8 \mathrm{~A}$ resolution. Nature 1997, 389:251-260.

4. Allfrey VG, Faulkner R, Mirsky AE: Acetylation and methylation of histones and their possible role in the regulation of rna synthesis. Proc Natl Acad Sci USA 1964, 51:786-794.

5. Berger SL: Histone modifications in transcriptional regulation. Curr Opin Genet Dev 2002, 12:142-148.

6. Kouzarides T: Chromatin modifications and their function. Cell 2007, 128:693-705.

7. Marks PA, Miller T, Richon VM: Histone deacetylases. Curr Opin Pharmacol 2003, 3:344-351. 
8. Ma P, Schultz RM: Histone deacetylase 1 (HDAC1) regulates histone acetylation, development, and gene expression in preimplantation mouse embryos. Dev Biol 2008, 319:110-120.

9. Aoki F, Worrad DM, Schultz RM: Regulation of transcriptional activity during the first and second cell cycles in the preimplantation mouse embryo. Dev Biol 1997, 181:296-307.

10. Strahl $B D$, Allis CD: The language of covalent histone modifications. Nature 2000, 403:41-45.

11. Turner BM: Histone acetylation and an epigenetic code. Bioessays 2000, 22:836-845.

12. VerMilyea MD, O'Neill LP, Turner BM: Transcription-independent heritability of induced histone modifications in the mouse preimplantation embryo. PLoS One 2009, 4:e6086.

13. Wang Y, Wysocka J, Sayegh J, Lee YH, Perlin JR, Leonelli L, Sonbuchner LS, McDonald CH, Cook RG, Dou Y, et al: Human PAD4 regulates histone arginine methylation levels via demethylimination. Science 2004, 306:279-283.

14. Li P, Yao H, Zhang Z, Li M, Luo Y, Thompson PR, Gilmour DS, Wang Y: Regulation of $\mathrm{p} 53$ target gene expression by peptidylarginine deiminase 4 . Mol Cell Biol 2008, 28:4745-4758.

15. Yao H, Li P, Venters BJ, Zheng S, Thompson PR, Pugh BF, Wang Y: Histone Arg modifications and p53 regulate the expression of OKL38, a mediator of apoptosis. J Biol Chem 2008, 283:20060-20068.

16. Cherrington BD, Morency E, Struble AM, Coonrod SA, Wakshlag JJ: Potential role for peptidylarginine deiminase 2 (PAD2) in citrullination of canine mammary epithelial cell histones. PLoS One 2010, 5:e11768.

17. Esposito G, Vitale AM, Leijten FP, Strik AM, Koonen-Reemst AM, Yurttas P, Robben TJ, Coonrod S, Gossen JA: Peptidylarginine deiminase (PAD) 6 is essential for oocyte cytoskeletal sheet formation and female fertility. Mol Cell Endocrinol 2007, 273:25-31.

18. Kim B, Kan R, Anguish L, Nelson LM, Coonrod SA: Potential role for MATER in cytoplasmic lattice formation in murine oocytes. PLoS One 2010, 5:e12587.

19. Cermelli S, Guo Y, Gross SP, Welte MA: The lipid-droplet proteome reveals that droplets are a protein-storage depot. Curr Biol 2006, 16:1783-1795.

20. Slack JL, Causey CP, Thompson PR: Protein arginine deiminase 4: a target for an epigenetic cancer therapy. Cell Mol Life Sci 2011, 68:709-720.

21. Chumanevich AA, Causey CP, Knuckley BA, Jones JE, Poudyal D, Chumanevich AP, Davis T, Matesic LE, Thompson PR, Hofseth L: Suppression of colitis in mice by Cl-amidine: a novel peptidylarginine deiminase inhibitor. Am J Physiol Gastrointest Liver Physiol 2011, 300:G929-G938.

22. Willis VC, Gizinski AM, Banda NK, Causey CP, Knuckley B, Cordova KN, Luo Y, Levitt B, Glogowska M, Chandra P, et al: N-alpha-benzoyl-N5(2-chloro-1-iminoethyl)-L-ornithine amide, a protein arginine deiminase inhibitor, reduces the severity of murine collageninduced arthritis. J Immunol 2011, 186:4396-4404.

23. Li P, Hu J, Wang Y: Methods for analyzing histone citrullination in chromatin structure and gene regulation. Methods Mol Biol 2012, 809:473-488.

24. Liu H, Kim JM, Aoki F: Regulation of histone H3 lysine 9 methylation in oocytes and early pre-implantation embryos. Development 2004, 131:2269-2280.

25. Tong ZB, Gold L, Pfeifer KE, Dorward H, Lee E, Bondy CA, Dean J, Nelson LM: Mater, a maternal effect gene required for early embryonic development in mice. Nat Genet 2000, 26:267-268.

26. Kan R, Yurttas P, Kim B, Jin M, Wo L, Lee B, Gosden R, Coonrod SA Regulation of mouse oocyte microtubule and organelle dynamics by PADI6 and the cytoplasmic lattices. Dev Biol 2011, 350:311-322.

27. Kito S, Hayao T, Noguchi-Kawasaki Y, Ohta Y, Hideki U, Tateno S: Improved in vitro fertilization and development by use of modified human tubal fluid and applicability of pronucleate embryos for cryopreservation by rapid freezing in inbred mice. Comp Med 2004, 54:564-570.

doi:10.1186/1471-213X-12-19

Cite this article as: Kan et al.: Potential role for PADI-mediated histone citrullination in preimplantation development. BMC Developmental Biology 2012 12:19.

\section{Submit your next manuscript to BioMed Central and take full advantage of:}

- Convenient online submission

- Thorough peer review

- No space constraints or color figure charges

- Immediate publication on acceptance

- Inclusion in PubMed, CAS, Scopus and Google Scholar

- Research which is freely available for redistribution 